\title{
Efecto del nopal (Opuntia spp) deshidratado en polvo sobre las propiedades fisicoquímicas y sensoriales de salchichas Viena
}

\author{
Effect of dehydrated nopal (Opuntia spp) powder on physicochemical and sensory properties \\ of Vienna sausages
}

\begin{abstract}
Lizbeth Malleli Diego-Zarate ${ }^{1}$, Gerardo Méndez-Zamora ${ }^{2}$, Jocelyn Abigail Rivera-De Alba ${ }^{3}$, Emmanuel Flores-Girón ${ }^{1 *}$ Universidad Autónoma Chapingo, Departamento de Ingeniería Agroindustrial, Carr. Federal México-Texcoco km 38.5, CP. 56230, Texcoco, Estado de México, México.

2 Universidad Autónoma de Nuevo León, Facultad de Agronomía, Francisco Villa s/n, Ex-Hacienda El Canadá, 66050, General Escobedo, Nuevo León, México.

Unidad Profesional Interdisciplinaria de Biotecnología, Instituto Politécnico Nacional, Unidad Profesional Interdisciplinaria de Biotecnología, Instituto Politécnico Nacional, Av. Acueducto s/n, Col. La Laguna Ticomán, 07340 G.A.M Ciudad de México, México.
\end{abstract}

\section{RESUMEN}

Los cladodios de nopal (Opuntia spp) son estructuras vegetales con alto contenido de fibra dietaria, ingrediente clave para el actual desarrollo de alimentos cárnicos funcionales; sin embargo, su incorporación en productos emulsificados, como las salchichas, repercute en sus propiedades físicas, químicas y sensoriales. Por ello, en el presente trabajo se estudiaron los efectos de la adición de nopal deshidratado en polvo sobre la composición química, las pérdidas por cocción, la capacidad de retención de agua, el color, el perfil de textura y los atributos sensoriales de salchichas Viena en concentraciones de $0 \%$ (TNOPO), $2 \%$ (TNOP2), $4 \%$ (TNOP4) y $6 \%$ (TNOP6). La incorporación de este ingrediente redujo el contenido de grasa al aumentar el contenido de fibra cruda, a la vez que afectó su apariencia disminuyendo su luminosidad y color rojo e incrementado el amarillo. La pérdida por cocción y la capacidad de retención de agua no se vieron afectadas, pero sí el perfil de textura cuando el nivel de incorporación fue del $6 \%$; mientras que, más del $4 \%$ disminuyó la aceptabilidad global y por atributo. Una adición del $2 \%$ de harina de nopal puede emplearse en el desarrollo de productos cárnicos funcionales emulsificados.

Palabras clave: Pastas cárnicas, fibra dietética, textura, color.

\section{ABSTRACT}

Nopal cladodes (Opuntia spp) are vegetal structures with high dietary fiber content, a key ingredient for the current development of functional meat foods; nevertheless, its addition in emulsified products, like sausages, affects its physical, chemical and sensory properties. Thus, in the present work the effects of the inclusion of dehydrated nopal powder in chemical composition, cooking losses, water holding capacity, texture profile and sensory attributes of Vienna sausages were analyzed at concentrations of $0 \%$ (TNOP0), 2 $\%$ (TNOP2), $4 \%$ (TNOP4) and $6 \%$ (TNOP6). The incorporation of this ingredient reduced fat content by increasing crude fiber, at the same time that affected appereance by decreasing luminosity and red color and enhancing yellow. The cooking loss and water holding capacity were not affected, but yes texture profile when the addition level was $6 \%$; while more

*Autor para correspondencia: Emmanuel Flores Girón

Correo electrónico: efloresg@chapingo.mx

Recibido: 22 de septiembre de 2020

Aceptado: 25 de enero de 2021 than $4 \%$ reduced global and by attribute acceptance. The addition of $2 \%$ nopal flour is feasible in emulsified functional meat products development.

Keywords: Meat batters, dietary fiber, texture, color.

\section{INTRODUCCIÓN}

Frecuentemente, los productos cárnicos son señalados por causar enfermedades cardiovasculares, algunos tipos de cáncer y obesidad, debido a su contenido de grasa saturada y colesterol (Ospina-Meneses et al., 2011). En las últimas décadas la industria alimentaria se ha preocupado por la formulación y desarrollo de nuevos productos que están dirigidos a aportar alternativas más saludables a aquellas personas que los consumen.

La fibra dietética es uno de los componentes más estudiados desde el punto de vista nutrimental. De acuerdo con su naturaleza, presenta numerosas características funcionales que incluyen la capacidad de retención de agua (CRA), lubricación, estabilidad de congelación/descongelación y modificación de la textura, por lo cual, la fibra es un extendedor, aglutinante y un ingrediente de reemplazo de grasa valioso en la fabricación de diversos productos alimenticios (Talukder, 2015). En el desarrollo de productos cárnicos, se han probado varias fuentes de fibra como avena, remolacha azucarera, soja, guisantes, psyllium, cáscara de tuna etc. en la formulación de hamburguesas y salchichas para mejorar su calidad nutricional, así como sus propiedades fisicoquímicas y sensoriales (Díaz-Vela et al., 2017; Mehta et al., 2015).

El nopal es una planta de la familia Cactaceae de los géneros Opuntia y Nopalea que se produce principalmente en zonas templadas, semiáridas y tropicales secas de México; diferentes partes de la planta son de interés para su industrialización, por ejemplo, el fruto (conocido como tuna) como parte comestible, y los cladodios para la obtención de fibras y la producción de harinas (Saenz et al., 2006; Sánchez-Bell, 2006).

El nopal es una fuente importante de fibra dietética. Según su solubilidad en agua, la fibra se clasifica en soluble e insoluble; la primera la conforman mucílagos, gomas, pectinas y hemicelulosas, mientras que la insoluble es princi- 
palmente celulosa, lignina y una gran fracción de hemicelulosa (Saenz, 1997; Saenz et al., 2012). Estas fracciones de fibra tienen efectos fisiológicos distintos: la fibra soluble se asocia con la reducción de los niveles de glucosa y de colesterol y la estabilización del vaciamiento gástrico; la fibra insoluble, por su parte, se relaciona con la capacidad de retención de agua (aumento del peso de las heces), el intercambio iónico, la absorción de ácidos biliares, minerales, vitaminas y otros, así como su interacción con la flora microbiana (Saenz et al., 2006).

De acuerdo con Saenz et al. (2006) y Bautista-Justo et al. (2010), los cladodios, tallos o pencas del nopal son una fuente importante de fibra, mucílagos y calcio, tres componentes que son necesarios para integrar una dieta saludable. Además, Rocchetti et al. (2018) mencionaron que los cladodios de nopal poseen actividades antidiabéticas y antioxidantes, las cuales se han atribuido a cantidades significativas de polisacáridos, lignanos, flavonoles y ácidos fenólicos. Los cladodios también contienen ácido palmitelaídico (C16:1 trans-9), un ácido graso monoinsaturado que regula el metabolismo de los ácidos grasos para su aplicación en el control del peso (Kadegowda et al., 2013; Aruwa et al., 2018). Debido al potencial nutricional y funcional que la composición del nopal ostenta, el presente trabajo tuvo como objetivo estudiar los cambios en las características fisicoquímicas y sensoriales de salchichas Viena causados por la incorporación de nopal deshidratado en polvo.

\section{MATERIALES Y MÉTODOS}

Como fuente de fibra para los diferentes tratamientos se utilizó nopal deshidratado en polvo (NOP) de la marca comercial Natura Bio Foods. Se determinaron su contenido de humedad, proteína, extracto etéreo y cenizas con base en los métodos de la AOAC (1998), y su contenido de fibra cruda por el método de Van de Kamer y Van Ginkel (1952).

\section{Tratamientos y elaboración de la pasta cárnica tipo sal- chicha Viena}

Se formularon cuatro tratamientos; TNOPO: testigo (sin nopal deshidratado en polvo), TNOP2: $2 \%$ de nopal deshidratado en polvo, TNOP4: $4 \%$ de nopal deshidratado en polvo, y TNOP6: $6 \%$ de nopal deshidratado en polvo. La formulación utilizada para la elaboración de las salchichas se muestra en la Tabla 1. La elaboración de las salchichas se realizó de acuerdo con el proceso propuesto por Méndez-Zamora et al. (2015). Se empleó carne de res y de cerdo parcialmente congelada, misma que fue picada con una cortadora-picadora de carne (HOBART ${ }^{\oplus}$ cutter, Hobart Corporation, Modelo 84145 , USA) por $3 \mathrm{~min}$, mientras se incorporaban las sales de cura, el eritorbato y un tercio del hielo, lentamente. Después, se agregaron los polifosfatos y un tercio del hielo, y se siguió moliendo por $2 \mathrm{~min}$. Luego, se incorporaron el nopal deshidratado en polvo junto con los condimentos y el proceso de formación de la pasta cárnica continuó por 3 min, cuidando que la temperatura no superara los $11^{\circ} \mathrm{C}$; se añadió la grasa de cerdo parcialmente congelada y se molió 2 min. Finalmen-
Tabla 1. Formulación de pastas cárnicas preparadas con nopal deshidratado en polvo.

Table 1. Formulation of meat batters prepared with dehydrated nopal powder.

\begin{tabular}{lcccc}
\hline \multirow{2}{*}{ Ingredientes (\%) } & \multicolumn{4}{c}{ Tratamientos $^{1}$} \\
\cline { 2 - 5 } & TNOPO & TNOP2 & TNOP4 & TNOP6 \\
\hline Carne de res & 39 & 39 & 39 & 39 \\
Carne de cerdo & 17 & 17 & 17 & 17 \\
\hline Lardo de cerdo & 14 & 14 & 14 & 14 \\
\hline Hielo & 30 & 30 & 30 & 30 \\
\hline Total & 100 & 100 & 100 & 100 \\
\hline Condimentos & 2.4 & 2.4 & 2.4 & 2.4 \\
\hline Sal & 2.0 & 2.0 & 2.0 & 2.0 \\
\hline Fosfatos & 0.39 & 0.39 & 0.39 & 0.39 \\
\hline Eritorbato de sodio & 0.05 & 0.05 & 0.05 & 0.05 \\
\hline Glutamato monosódico & 0.05 & 0.05 & 0.05 & 0.05 \\
\hline Almidón & 5.92 & 5.92 & 5.92 & 5.92 \\
Nopal deshidratado en polvo & 0.00 & 2 & 4 & 6 \\
\hline
\end{tabular}

1 TNOPO: testigo (sin nopal deshidratado en polvo), TNOP2: $2 \%$ de nopal deshidratado en polvo, TNOP4: $4 \%$ de nopal deshidratado en polvo, TNOP6: $6 \%$ de nopal deshidratado en polvo.

te, se añadieron el almidón y el resto del hielo prolongando la molienda 3 min más. Una vez obtenida la pasta cárnica fue embutida en funda de celulosa de $2 \mathrm{~cm}$ de diámetro con una embutidora vertical manual (eemb3, Bonne Concasse, México). Las salchichas fueron atadas manualmente con hilo cada $12 \mathrm{~cm}$. Previo a la cocción, las salchichas se envolvieron con una película de polietileno; el tratamiento térmico, en baño de agua, terminó al alcanzar $70{ }^{\circ} \mathrm{C}$ en el centro de las salchichas. Posteriormente, las salchichas se enfriaron en un baño de agua y hielo a $4{ }^{\circ} \mathrm{C}$ por $20 \mathrm{~min}$, se escurrieron por 10 min y se refrigeraron a $4{ }^{\circ} \mathrm{C}$ en bolsas de polietileno hasta su análisis. Los tratamientos se realizaron por triplicado, cada réplica representó un bloque, que fue la elaboración de los cuatro tratamientos en tiempos diferentes.

\section{Pérdida por cocción y capacidad de retención de agua (CRA)}

Las pérdidas por cocción se evaluaron a las 24 h (día 0 ) a partir del peso de las salchichas cocidas menos el peso de las salchichas crudas, según lo recomendado por SilvaVazquez et al. (2018). La CRA se evaluó en el día 7 mediante el método sugerido por Dzudie et al. (2005) y modificado por Méndez-Zamora et al. (2015); para lo cual, aproximadamente $0.3 \mathrm{~g}$ de muestra de salchicha se colocaron entre dos papeles filtro y estos, a su vez, entre dos placas de acrílico de $12 \times 12$ $\mathrm{cm}$ a las cuales se les aplicó una fuerza de compresión de $4 \mathrm{~kg}$ durante $20 \mathrm{~min}$. Se consideró que, debido a la compresión, el agua libre migró desde la muestra hacia el papel y se calculó el \% de agua libre y CRA con base en las ecuaciones 1 y 2.

$\%$ de agua libre $=\left[\left(M_{i}-M_{f}\right) / M_{i}\right] * 100$

CRA $=100$ - \% de agua libre

Donde $M_{i}$ es el peso inicial de la muestra y $M_{f}$ el peso final.

\section{Análisis proximal de las salchichas}

Al día 7, después de la elaboración de cada unidad experimental, se determinó su composición química me- 
diante los procedimientos $950.46,985.15,992.15$ y 920.153 de la AOAC (1998) para humedad, grasa, proteína y cenizas, respectivamente; $y$ el contenido de fibra cruda por el método Van de Kamer y Van Ginkel (1952). Todas las evaluaciones se realizaron por triplicado.

\section{Análisis de color}

El color se evaluó directamente en la parte interna de las salchichas al día 7 después de la elaboración. Se utilizó un colorímetro Hunter Lab (Xe Plus, HunterLab, USA), calibrado con una placa blanca. Las lecturas se realizaron por triplicado para registrar los parámetros del sistema $\mathrm{CIE}\left(L^{*}, a^{*}\right.$ y b*). Adicionalmente, se calcularon el índice de color (IC), el ángulo de matiz o tono, el croma y el cambio total de color $(\Delta \mathrm{E})$, con base en lo propuesto por Mathias y Ah (2014) y Silva-Vazquez et al. (2018).

\section{Análisis de perfil de textura instrumental (APT)}

El APT se realizó al día 7 después de la elaboración de las salchichas utilizando un texturómetro TAXT2i (Stable Micro Systems, UK) con una celda de $5 \mathrm{~kg}$, y una sonda cilíndrica de aluminio P50 ( $50 \mathrm{~mm}$ de diámetro). Las salchichas fueron cortadas en cilindros de $15 \mathrm{~mm}$ de altura, y se realizaron cinco lecturas por cada tratamiento/réplica. Se emplearon las velocidades recomendadas por Zhao et al. (2018): $2 \mathrm{~mm} / \mathrm{s}$ para pre-ensayo y ensayo, y $5 \mathrm{~mm} / \mathrm{s}$ post-ensayo; una deformación de $40 \%$ y dos ciclos de compresiones se llevaron a cabo en cada prueba. Los parámetros evaluados fueron dureza $(\mathrm{N})$, cohesividad (adimensional), elasticidad ( $\mathrm{mm})$, gomosidad (N) y masticabilidad ( $\mathrm{N} \mathrm{mm}$ ) de acuerdo con Bourne (1978), Abbasi et al. (2019) y Zhao et al. (2018).

\section{Evaluación sensorial}

Se realizó una prueba afectiva con 90 panelistas semi entrenados, consumidores habituales de salchichas, para los tratamientos que presentaron mejores resultados en el análisis de perfil de textura: TNOP0, TNOP2 y TNOP4. Se empleó una escala hedónica de nueve puntos donde: $9=$ me gusta extremadamente, y 1 = me disgusta extremadamente (Meilgaard et al., 2006); se evaluó el nivel de agrado por atributo (apariencia, color, firmeza, sabor y sabor residual) y la aceptabilidad global. Se asignó un código de tres dígitos, diferente para cada muestra; cada panelista evaluó las tres muestras en orden aleatorio. Los datos obtenidos se analizaron mediante un diseño de bloques completamente al azar.

\section{Análisis estadístico}

Se empleó un arreglo de datos mediante un diseño de bloques completamente al azar y se realizó un ANOVA considerando como fuentes de variación a los tratamientos (\% de nopal deshidratado en polvo) y a los bloques (lotes de carnes empleados y fecha de elaboración) en el modelo estadístico. Cuando existieron diferencias significativas la hipótesis nula fue rechazada $(p<0.05)$ y se realizó la prueba estadística Tukey $(a=0.05)$ para la comparación de medias. El análisis de datos se realizó con Minitab ${ }^{\circledR}$ 17.1.0 (Minitab Inc. 2003) mediante el modelo lineal general.

\section{RESULTADOS Y DISCUSIÓN}

El nopal deshidratado en polvo presentó un $92.5 \%$ de sólidos, siendo carbohidratos $48.69 \%$, cenizas $19.14 \%$, proteínas $17.83 \%$, fibra cruda $14.31 \%$ y grasas $0.03 \%$. Esta composición es similar a la reportada por Pimienta (1990) para nopales jóvenes con edades entre uno y dos años.

\section{Pérdida por cocción y CRA}

La pérdida por cocción es un parámetro importante en los alimentos tratados térmicamente, ya que indica el rendimiento en el proceso. Las pérdidas por cocción de los tratamientos oscilaron entre 0.6 y $1.7 \%$, sin obtener diferencias significativas entre tratamientos $(p>0.05)$. Sin embargo, otros autores han demostrado que la incorporación de ingredientes como inulina y pectina pueden mejorar el rendimiento (Méndez-Zamora et al., 2015) y, de acuerdo con Díaz-Vela et al. (2015), la incorporación de harina de cáscara de tuna puede mejorar el rendimiento en la elaboración de salchichas cocidas inoculadas con bacterias ácido-lácticas.

En este trabajo, la CRA fue similar $(p>0.05)$ entre los tratamientos, obteniendo valores de 70.8 a $73.6 \%$. En otros estudios, Silva-Vazquez et al. (2018) y Méndez-Zamora et al. (2015) encontraron un aumento en la CRA al incorporar inulina y pectina; mientras que, Díaz-Vela et al. (2015) reportaron que la harina de cáscara de tuna brinda una mayor CRA en productos cárnicos debido a su contenido de fibra dietaria. Por otro lado, Hleap-Zapata et al. (2017) concluyeron que al reemplazar la harina de trigo por harina de quinua disminuye la CRA, lo que sugiere que la incorporación de ingredientes puede modificar la CRA del producto, aunque el comportamiento dependerá de la naturaleza del ingrediente añadido.

\section{Análisis proximal}

La composición general de los productos cárnicos puede cambiar con la incorporación de fibra. En la Tabla 2 se muestran los resultados del análisis proximal de los diferentes tratamientos. En la humedad no se obtuvieron diferencias significativas $(p>0.05)$ entre tratamientos. A mayor incorporación de nopal deshidratado en polvo (TNOP0, TNOP2, TNOP4 y TNOP6) disminuyó el contenido de humedad. Este comportamiento también fue observado por Yadav et al. (2018), quienes señalaron que la disminución en el contenido de humedad de salchichas de pollo adicionadas con salvado de trigo y orujo de zanahoria seco se debe al reemplazo cuantitativo de la carne con fuentes de fibra dietética que contienen muy poca humedad (5.09\% a $9.47 \%)$ en comparación con la carne.

Para los sólidos, el contenido de cenizas incrementó ( $p$ $<0.05$ ) a mayor incorporación de nopal deshidratado; lo que podría deberse a que este ingrediente tiene un alto contenido de cenizas (19.14\%), conformadas principalmente por minerales como el calcio y el fósforo, según señalan BautistaJusto et al. (2010). Este incremento coincide con los resultados obtenidos por otros autores. Hleap-Zapata et al. (2017) encontraron un incremento en el contenido de cenizas de las 
Tabla 2. Composición química de salchichas Viena incorporadas con diferentes niveles de nopal deshidratado en polvo.

Table 2. Chemical composition of Vienna sausages added with different levels of dehydrated nopal powder.

\begin{tabular}{lcccccc}
\hline \multirow{2}{*}{ Tratamientos $^{1}$} & \multicolumn{5}{c}{ Características (\%) } \\
\cline { 2 - 7 } & Humedad & Cenizas & Proteína & Grasa & Fibra & CHOS \\
\hline TNOP0 & $68.91^{\mathrm{a}}$ & $8.25^{\mathrm{b}}$ & $33.46^{\mathrm{a}}$ & $35.64^{\mathrm{a}}$ & $0.84^{\mathrm{d}}$ & $21.81^{\mathrm{a}}$ \\
\hline TNOP2 & $68.75^{\mathrm{a}}$ & $8.76^{\mathrm{ab}}$ & $33.09^{\mathrm{a}}$ & $33.60^{\mathrm{ab}}$ & $1.25^{\mathrm{c}}$ & $23.31^{\mathrm{a}}$ \\
\hline TNOP4 & $67.41^{\mathrm{b}}$ & $9.16^{\mathrm{ab}}$ & $31.39^{\mathrm{a}}$ & $32.86^{\mathrm{b}}$ & $1.68^{\mathrm{b}}$ & $24.91^{\mathrm{a}}$ \\
TNOP6 & $66.58^{\mathrm{b}}$ & $9.37^{\mathrm{a}}$ & $30.89^{\mathrm{a}}$ & $31.94^{\mathrm{b}}$ & $1.92^{\mathrm{a}}$ & $25.88^{\mathrm{a}}$ \\
\hline valor $p$ & $0.001^{\mathrm{a}}$ & 0.044 & $0.366^{\circ}$ & 0.005 & 0.000 & 0.161 \\
\hline
\end{tabular}

${ }^{1}$ TNOPO: testigo (sin nopal deshidratado en polvo), TNOP2: $2 \%$ de nopal deshidratado en polvo, TNOP4: $4 \%$ de nopal deshidratado en polvo, TNOP6: $6 \%$ de nopal deshidratado en polvo. Medias en la misma columna con superíndices diferentes son significativamente diferentes $(p<0.05)$. Unidades experimentales por tratamiento $(n)=9$.

salchichas incorporadas con harina de quinua, mientras que Méndez-Zamora et al. (2015) encontraron un aumento en las cenizas al incorporar inulina y pectina.

En el contenido de grasa se observó un descenso ( $p$ $<0.05)$ a mayor contenido de nopal deshidratado, siendo TNOP6 (6\% nopal deshidratado en polvo) el tratamiento con menor contenido de grasa. Powell et al. (2019) también encontraron un descenso en el contenido de grasa de las salchichas al incorporar $1.0 \%$ de fibra de cítricos. Este patrón es esperado considerando que los ingredientes incorporados como fuente de fibra son carentes de grasas.

El contenido de carbohidratos y proteínas de los distintos tratamientos no mostró diferencias significativas ( $p$ $>0.05) ;$ no así en el contenido de fibra cruda, donde hubo diferencias significativas $(p<0.05)$ entre todos los tratamientos. El contenido de fibra cruda en las salchichas aumentó conforme aumentó el nivel de incorporación de nopal deshidratado (TNOP0, TNOP2, TNOP4 y TNOP6). El tratamiento TNOP6 mostró un contenido de fibra cruda promedio de 1.92 $\%$ mientras que el TNOP4 ( $4 \%$ nopal deshidratado en polvo) obtuvo un promedio de $1.68 \%$, lo que podría mejorar el perfil nutricional de estos alimentos como ha sido señalado por Mehta et al. (2018), pues la incorporación de fibra generalmente reduce el contenido de grasa, aumenta la fracción de carbohidratos y fibra dietética, generando una nueva clase de productos cárnicos funcionales con mayores beneficios para la salud.

\section{Análisis de color}

La Tabla 3 muestra los resultados obtenidos para los parámetros de color. La luminosidad $\left(L^{*}\right)$ tuvo cambios significativos $(p<0.05)$ entre los diferentes tratamientos. Se observó un descenso de $L^{*}$ a medida que se incrementó el porcentaje de nopal deshidratado; la luminosidad pasó de 57.19 a 51.82, indicando así un oscurecimiento que afectó la apariencia de las salchichas. Las medias de $a^{*}$ no mostraron diferencias significativas $(p>0.05)$ entre tratamientos, pero se observó un ligero descenso a medida que incrementó
Tabla 3. Efecto de la incorporación de nopal deshidratado en el color de salchichas Viena.

Table 3. Influence of dehydrated nopal powder addition on Vienna sausages color.

\begin{tabular}{lcccccccc}
\hline & \multicolumn{8}{c}{ Variables de color $^{2}$} \\
\cline { 2 - 9 } Tratamientos $^{1}$ & $\boldsymbol{L}^{*}$ & $\boldsymbol{a}^{*}$ & $\boldsymbol{b}^{*}$ & $\begin{array}{c}\text { Ángulo } \\
\text { de } \\
\text { matiz }\end{array}$ & Croma & $\boldsymbol{\Delta E}$ & IC \\
\hline TNOP0 & $57.19^{\mathrm{a}}$ & $11.09^{\mathrm{a}}$ & $10.32^{\mathrm{c}}$ & $42.31^{\mathrm{c}}$ & $15.19^{\mathrm{a}}$ & $29.86^{\mathrm{b}}$ & $33.27^{\mathrm{b}}$ \\
\hline TNOP2 & $53.55^{\mathrm{ab}}$ & $11.26^{\mathrm{a}}$ & $12.05^{\mathrm{bc}}$ & $47.27^{\mathrm{bc}}$ & $16.57^{\mathrm{a}}$ & $33.70^{\mathrm{ab}}$ & $39.58^{\mathrm{ab}}$ \\
\hline TNOP4 & $52.49^{\mathrm{b}}$ & $10.69^{\mathrm{a}}$ & $13.11^{\mathrm{ba}}$ & $51.59^{\mathrm{ab}}$ & $17.03^{\mathrm{a}}$ & $34.80^{\mathrm{a}}$ & $42.5^{\mathrm{a}}$ \\
\hline TNOP6 & $51.82^{\mathrm{b}}$ & $9.44^{\mathrm{a}}$ & $14.73^{\mathrm{a}}$ & $57.68^{\mathrm{a}}$ & $17.55^{\mathrm{a}}$ & $35.57^{\mathrm{a}}$ & $45.56^{\mathrm{a}}$ \\
\hline valor $p$ & 0.01 & 0.383 & 0.001 & 0.002 & 0.226 & 0.015 & 0.013 \\
\hline
\end{tabular}

${ }^{1}$ TNOP0: testigo (sin nopal deshidratado en polvo), TNOP2: $2 \%$ de nopal deshidratado en polvo, TNOP4: $4 \%$ de nopal deshidratado en polvo, TNOP6: $6 \%$ de nopal deshidratado en polvo.

${ }^{2} L^{*}$ : luminosidad, $a^{*}$ : tendencia al color rojo, $b^{*}$ : tendencia al color amarillo, $\Delta \mathrm{E}$ : cambio total de color, IC: índice de coloración. Medias en la misma columna con superíndices diferentes son significativamente diferentes $(p<$ 0.05). Unidades experimentales por tratamiento $(n)=9$.

el porcentaje de nopal deshidratado, lo que señala que los tratamientos con mayor contenido de nopal muestran una leve reducción del rojo. Las medias de $b^{*}$ entre los diferentes tratamientos muestran diferencias significativas $(p<0.05)$, se observó un aumento en los valores de $b^{*}$, de 10.32 en el tratamiento testigo (TNOP0) a 14.73 en el tratamiento TNOP6, lo cual mostró que, al aumentar el contenido de nopal deshidratado, el color de las muestras se inclina hacia el amarillo.

En este estudio, los valores $L^{*}, a^{*}, b^{*}$, ángulo de matiz y croma indicaron que la adición de nopal deshidratado en polvo modifica el color de las salchichas posiblemente debido a la clorofila presente en los nopales. La clorofila es el pigmento responsable del característico color verde de las frutas y verduras, es altamente susceptible a la degradación durante el procesamiento, lo que resulta en cambios de color en los alimentos (Bautista et al., 2016). Durante el proceso térmico el átomo de magnesio central del anillo de porfirina de la clorofila es reemplazado por dos átomos de hidrógeno para formar feofitina, que se acompaña de un cambio de color indeseable de verde brillante a marrón oliva. Dado que el color es una característica sensorial importante para determinar la aceptabilidad del producto, es de vital importancia para la industria alimentaria prevenir o al menos minimizar la degradación de la clorofila durante el procesamiento (Weemaes et al., 1999). Respecto al ángulo de matiz se encontraron diferencias significativas $(p<0.05)$ entre tratamientos; el tratamiento con el ángulo de matiz menor fue TNOPO mientras que el mayor fue TNOP6, mostrando que la incorporación de nopal deshidratado en polvo genera salchichas con coloraciones más cercanas hacia el amarillo que hacia el rojo.

Las medias de croma no mostraron diferencias significativas $(p>0.05)$ entre tratamientos, pero se observó un ligero incremento a medida que aumentó el porcentaje de nopal deshidratado. El aumento en los valores de croma indicó mayor saturación en los tratamientos que contienen nopal causando su oscurecimiento. 
El cambio de color total $(\Delta \mathrm{E})$ presentó diferencias significativas $(p<0.05)$ entre tratamientos. La incorporación de nopal deshidratado incrementó el $\Delta \mathrm{E}$ de las salchichas, mostrando que las diferencias de color son más evidentes a mayor nivel de incorporación de nopal (TNOPO-TNOP6). En cuanto al índice de color (IC) se encontraron diferencias significativas $(p<0.05)$ entre tratamientos; la incorporación de nopal aumentó significativamente este parámetro debido al alto contenido de pigmentos. El aumento en los valores del croma, $\Delta \mathrm{E}$ e IC coinciden con los datos reportados por Silva-Vazquez et al. (2018), no obstante, el ángulo de matiz difiere debido a que la inulina y pectina utilizados para el estudio eran de color blanco, mientras que el nopal tiene una coloración amarilla-verdosa, promoviendo que la coloración de las salchichas tienda hacia el color amarillo, con una disminución en el rojo y la saturación que provocaron su oscurecimiento, resultando en un posible impacto negativo para la aceptación del producto.

\section{Análisis de perfil de textura instrumental}

Se encontraron cambios $(p<0.05)$ en las propiedades texturales de las salchichas incorporadas con nopal deshidratado (Tabla 4). La dureza de los tratamientos aumentó a medida que se incorporó hasta un $4 \%$ de nopal deshidratado (TNOP4). En cambio, el tratamiento con $6 \%$ de nopal (TNOP6) mostró un descenso del $43 \%$ respecto al tratamiento testigo (TNOP0). Otros estudios indicaron que incorporar fibras a la formulación de salchichas modifica sus propiedades texturales en función del tipo de fibra y porcentaje de incorporación. Por ejemplo, Flores et al. (2005) reportaron un incremento de dureza en las salchichas cuando incorporaron fibra de avena, pero una disminución cuando se incorporó más de $1 \%$ de fibra de trigo. Diferentes autores han realizado estudios para evaluar el uso de diferentes fibras como reemplazante de ingredientes como la grasa y los fosfatos. Powell et al. (2019) evaluaron la adición de fibra de cítricos en salchichas Bologna como sustituto natural de fosfato de sodio $(0.50,0.75 \mathrm{y}$ $1.0 \%)$ y también encontraron un aumento en la dureza a mayor concentración de fibra. Zhao et al. (2018) coinciden en que, a mayor concentración de fibra de celulosa, la dureza se incrementa (testigo $=34.35 \mathrm{~N}$, tratamiento con $1.2 \%$ de fibra = $37.84 \mathrm{~N}$ ); igualmente observaron que la adición de agua como reemplazo de grasa podría dar lugar a una textura más suave, provocando un descenso en la dureza de las salchichas con reducción de grasa y sin adición de fibra. La naturaleza de la fibra incorporada influye en los cambios texturales observados. Zhao et al. (2018) encontraron un aumento en la dureza de salchichas incorporadas con fibra de celulosa regenerada; esta fibra tiene la capacidad de gelificar formando redes tridimensionales que modifican las propiedades reológicas de la fase continua de la emulsión, esto depende de la cantidad de grasa que contenga el producto.

En el presente estudio la incorporación de nopal deshidratado hasta en un $4 \%$ incrementó la dureza de las salchichas, posiblemente por el contenido de polisacáridos que gelifican con calor, como la hemicelulosa, aunque incorporar
Tabla 4. Efecto de la incorporación de nopal deshidratado sobre el perfil de textura instrumental (APT) de salchichas Viena.

Table 4. Effect of dehydrated nopal powder addition on Vienna sausages instrumental texture profile analysis (TPA).

\begin{tabular}{|c|c|c|c|c|c|}
\hline Tratamientos ${ }^{1}$ & $\begin{array}{c}\text { Dureza } \\
\text { (N) }\end{array}$ & Cohesividad & $\begin{array}{c}\text { Elasticidad } \\
(\mathrm{mm})\end{array}$ & $\begin{array}{c}\text { Gomosidad } \\
\text { (N) }\end{array}$ & $\begin{array}{c}\begin{array}{c}\text { Masticabilidad } \\
(\mathrm{N} \mathrm{mm})\end{array} \\
\end{array}$ \\
\hline TNOPO & $29.73^{b}$ & $0.83^{a}$ & $0.74^{\mathrm{a}}$ & $24.76^{b}$ & $17.69^{a}$ \\
\hline TNOP2 & $32.34^{a}$ & $0.84^{a}$ & $0.73^{a}$ & $26.86^{a b}$ & $18.83^{a}$ \\
\hline TNOP4 & $34.13^{a}$ & $0.80^{\mathrm{a}}$ & $0.71^{\mathrm{a}}$ & $27.63^{a}$ & $18.36^{a}$ \\
\hline TNOP6 & $16.84^{c}$ & $0.65^{\mathrm{b}}$ & $0.70^{\mathrm{a}}$ & $12.18^{c}$ & $7.73^{b}$ \\
\hline valor $p$ & 0.000 & 0.000 & 0.146 & 0.000 & 0.000 \\
\hline
\end{tabular}

${ }^{1}$ TNOP0: testigo (sin nopal deshidratado en polvo), TNOP2: $2 \%$ de nopal deshidratado en polvo, TNOP4: $4 \%$ de nopal deshidratado en polvo, TNOP6: $6 \%$ de nopal deshidratado en polvo. Medias en la misma columna con superíndices diferentes son significativamente diferentes $(p<0.05)$. Unidades experimentales por tratamiento $(n)=15$.

un $6 \%$ de nopal trajo consecuencias negativas para este parámetro. Esta observación coincide con el planteamiento de Flores et al. (2005) quienes sugieren que existe un punto de equilibrio entre los ingredientes en la pasta cárnica que permiten la incorporación de cierta cantidad de otro ingrediente.

La cohesividad de las salchichas disminuyó con la incorporación de nopal deshidratado, pero no se observaron diferencias significativas entre los tratamientos TNOP0, TNOP2 y TNOP4 El tratamiento TNOP6 mostró el valor más bajo de cohesividad con diferencia significativa $(p<0.05)$ respecto a los demás tratamientos. Este patrón de pérdida de cohesividad ha sido reportado por Powell et al. (2019) al incorporar fibra de cítricos en salchicha Bologna de cerdo, y por Zhao et al. (2018) al adicionar fibra de celulosa regenerada en salchichas bajas en grasa. La elasticidad, definida como la capacidad que tiene una muestra deformada para recuperar su forma o longitud inicial después de que una fuerza ha impactado en ella (Torres et al., 2015), no se vio afectada significativamente $(p>0.05)$ por la incorporación de nopal deshidratado.

En el parámetro de gomosidad se observó un incremento para los tratamientos TNOP2, TNOP4 con respecto al testigo (TNOP0), aunque el tratamiento con mayor cantidad de nopal incorporado (TNOP6) mostró un descenso significativo $(p<0.05)$. Para la masticabilidad no se encontraron diferencias significativas entre TNOP0, TNOP2 y TNOP4, pero sí con respecto de TNOP6 $(p<0.05)$, que mostró una pérdida significativa en este parámetro.

Méndez-Zamora et al. (2015) encontraron, en salchichas Frankfurt, que la gomosidad y la masticabilidad fueron menores en los tratamientos con fibras dietéticas, lo cual podría atribuirse al contenido de grasa y a la alta capacidad de unión y retención de agua de las fibras dietéticas. Adicionalmente, Powell et al. (2019) y Zhao et al. (2018) reportaron un aumento en la gomosidad y la masticabilidad de las salchichas al incorporar $1.0 \%$ de fibra de cítricos y $1.2 \%$ de fibra de celulosa regenerada, posiblemente debido a que la fibra de celulosa es altamente viscosa y tiene la capacidad de formar geles, confiriendo una apariencia y textura muy similares 
a la grasa. En el presente estudio, la pérdida de gomosidad y masticabilidad en el tratamiento TNOP6 puede asociarse a la concentración y naturaleza de los polisacáridos presentes en el nopal deshidratado. La incorporación de $6 \%$ de nopal deshidratado (TNOP6) afectó negativamente las propiedades texturales estudiadas, por lo que no fue considerado para la evaluación sensorial.

\section{Evaluación sensorial}

En la Tabla 5 se puede observar que las medias de aceptabilidad para los atributos de apariencia, color, sabor residual y aceptabilidad global de TNOPO y TNOP2 contra TNOP4 presentaron diferencias significativas $(p<0.05)$, mientras que entre TNOPO y TNOP2 no existió diferencia significativa $(p>0.05)$.

Se observaron diferencias significativas $(p<0.05)$ entre la aceptación del atributo de firmeza del tratamiento testigo (TNOPO) con respecto a los tratamientos incorporados con nopal deshidratado (TNOP2 y TNOP4), siendo el TNOP0 el más aceptado. Respecto al atributo sabor, se encontraron diferencias significativas $(p<0.05)$ entre los tres tratamientos; a medida que se incorporó mayor cantidad de nopal deshidratado, se observó un descenso en su aceptabilidad. Díaz-Vela et al. (2017) reportaron que la incorporación de harina de cáscara de tuna en salchichas modificó su color y, de acuerdo con la evaluación del panel, se percibieron más oscuras que el testigo; así mismo reportaron diferencias en la dureza y plasticidad.

Tabla 5. Evaluación sensorial de salchichas Viena incorporadas con nopal deshidratado en polvo.

Table 5. Sensory evaluation of Vienna sausages incorporated with dehydrated nopal powder.

\begin{tabular}{lcccccc}
\hline & \multicolumn{6}{c}{ Aceptabilidad por atributo } \\
\cline { 2 - 7 } Tratamiento & Apariencia & Color & Firmeza & Sabor & $\begin{array}{c}\text { Sabor } \\
\text { residual }\end{array}$ & $\begin{array}{c}\text { Aceptabilidad } \\
\text { global }\end{array}$ \\
\hline TNOP0 & $6.31^{\mathrm{a}}$ & $6.41^{\mathrm{a}}$ & $6.70^{\mathrm{a}}$ & $6.63^{\mathrm{a}}$ & $6.17^{\mathrm{a}}$ & $6.29^{\mathrm{a}}$ \\
TNOP2 & $6.16^{\mathrm{a}}$ & $6.11^{\mathrm{a}}$ & $6.13^{\mathrm{b}}$ & $5.86^{\mathrm{b}}$ & $5.76^{\mathrm{a}}$ & $6.01^{\mathrm{a}}$ \\
TNOP4 & $5.48^{\mathrm{b}}$ & $5.27^{\mathrm{b}}$ & $5.98^{\mathrm{b}}$ & $4.83^{\mathrm{c}}$ & $4.96^{\mathrm{b}}$ & $5.04^{\mathrm{b}}$ \\
valor $p$ & 0.000 & 0.000 & 0.000 & 0.000 & 0.000 & 0.000 \\
\hline
\end{tabular}

1 TNOPO: testigo (sin nopal deshidratado en polvo), TNOP2: $2 \%$ de nopal deshidratado en polvo, TNOP4: $4 \%$ de nopal deshidratado en polvo.

Medias en la misma columna con superíndices diferentes son significativamente diferentes $(p<0.05)$. Unidades experimentales por tratamiento $(n)$ $=90$.

De forma general, la incorporación de nopal deshidratado provocó efectos negativos en la aceptabilidad por atributo y global de las salchichas. Sin embargo, los tratamientos TNOP0 y TNOP2 mostraron una aceptación similar, por parte de los panelistas, para los atributos de apariencia, color, sabor residual y aceptabilidad global.

\section{CONCLUSIONES}

La incorporación de nopal deshidratado en la formulación de salchichas Viena disminuyó el contenido de grasa al aumentar el contenido de fibra cruda y generó una pérdida de luminosidad e incremento del color amarillo, sin alterar los parámetros de pérdida por cocción y capacidad de retención de agua.

La adición de $6 \%$ de nopal deshidratado redujo la dureza, cohesividad y gomosidad de las salchichas, mientras que más del $4 \%$ disminuyó la aceptabilidad global y por atributo. Es así que una adición del $2 \%$ de harina de nopal puede emplearse en el desarrollo de productos cárnicos emulsificados.

\section{REFERENCIAS}

Abbasi, E., Amini Sarteshnizi, R., Ahmadi Gavlighi, H., Nikoo, M., Azizi, M. H. y Sadeghinejad, N. 2019. Effect of partial replacement of fat with added water and tragacanth gum (Astragalus gossypinus and Astragalus compactus) on the physicochemical, texture, oxidative stability, and sensory property of reduced fat emulsion type sausage. Meat Science. 147: 135-143.

AOAC. 1998. Official methods of analysis of the AOAC International. 16th ed. Association of Official Analytical Chemistry International. Maryland, USA.

Aruwa, C. E., Amoo, S. O. y Kudanga, T. 2018. Opuntia (Cactaceae) plant compounds, biological activities and prospects - A comprehensive review. Food Research International. 112: 328-344.

Bautista, V., Núñez, A., Amaya, C., Báez, J., Espinoza, A., Rodríguez, A., Miranda, L., Castañeda, E. y Cárdena, M. 2016. Influencia del tipo y tiempo de cocción en la degradación de clorofila en hortalizas. Investigación y Desarrollo en Ciencia y Tecnología de Alimentos. 1(1): 411-416.

Bautista-Justo, M., Pineda-Torres, R.I., Camarena-Aguilar, E., Alanís-Guzmán, G., Da-Mota, V.M. y Barboza-Corona, J. E. 2010. El Nopal fresco como fuente de fibra y calcio en panqués. Acta Universitaria. 20: 11-17.

Bourne, M. C. 1978. Texture profile analysis. Food Technology: 32: 62-67.

Díaz-Vela, J., Totosaus, A. y Pérez-Chabela, M. L. 2015. Integration of Agroindustrial Co-Products as Functional Food Ingredients: Cactus Pear (Opuntia ficus indica) Flour and Pineapple (Ananas comosus) Peel Flour as Fiber Source in Cooked Sausages Inoculated with Lactic Acid Bacteria. Journal of Food Processing and Preservatio. 39: 2630-2638.

Díaz-Vela, J., Totosaus, A., Escalona-Buendía, H. B. y PérezChabela, M. L. 2017. Influence of the fiber from agroindustrial co-products as functional food ingredient on the acceptance, neophobia and sensory characteristics of cooked sausages. Journal of Food Science and Technology. 54: 379-385.

Dzudie , T., Scher, J. y Hardy, J. 2005. Effect of Fat Sources on the Physico-Chemical Nutritional and Textural Properties of Beef Sausages. Journal of Food Technology. 3: 220-225.

Flores, A., Burciaga, A., Soriano, C., Alonso M. y Ramírez, P. Uso de fibra de avena y trigo en salchicha Viena evaluando nivel de agrado y perfil de textura. [Consultado 7 de noviembre 2019] 2005. Disponible en: http://www.respyn.uanl.mx/ especiales/2005/ee132005/documentos/CNA23.pdf

Hleap-Zapata, J., Portillo, M. y Vera, J. 2017. Evaluación fisicoquímica y sensorial de salchichas con inclusión de harina de quinua (Chenopodium quinoa W.). Biotecnología en el Sector Agropecuario y Agroindustrial. 15, 61. 
Kadegowda, A. K. G., Burns, T. A., Miller, M. C. y Duckett, S. K. 2013. Cis-9, trans-11 conjugated linoleic acid is endogenously synthesized from palmitelaidic (C16:1 trans-9) acid in bovine adipocytes1. Journal of Animal Science, 91: 1614-1623.

Mathias, K. y Ah, K. 2014. El color en los alimentos un criterio de calidad medible. Agro Sur. 42: 40-46.

Mehta, N., Ahlawat, S., Sharma, D. y Dabur, R. 2015. Novel trends in development of dietary fiber rich meat products-a critical review. Journal of Food Science and Technology. 52(2): 633647.

Mehta, N., Kumar, M., Kumar, P., Prakash-Malav, O., Kumar, A. y Kumar, D. 2018. Development of Dietary Fiber-Rich Meat Products: Technological Advancements and Functional Significance. En: Bioactive Molecules in Food. J.M. Mérillon y K. Ramawat (ed.), pp 763-795. Springer Cham, Suiza.

Meilgaard, M., Civille, G. V. y Carr, T. B. 2006. Affective tests consumer tests and in-house panel acceptance tests. En: Sensory evaluation techniques. M. Meilgaard, G.V. Civille, y T.B. Carr (ed.), pp 307-360. CRC Press, USA.

Méndez-Zamora, G., García-Macías, J. A., Santellano-Estrada, E., Chávez-Martínez, A., Durán-Meléndez, L. A., Silva-Vázquez, R. y Quintero-Ramos, A. 2015. Fat reduction in the formulation of frankfurter sausages using inulin and pectin. Food Science and Technology (Campinas). 35: 25-31.

Ospina-Meneses, S. M., Restrepo-Molina, D. A. y López-Vargas, J. H. 2011. Derivados cárnicos como alimentos funcionales. Revista Lasallista de Investigación. 8:163-172.

Pimienta, E. 1990. El nopal tunero. Universidad de Guadalajara. Jalisco, México.

Powell, M. J., Sebranek, J. G., Prusa, K. J. y Tarté, R. 2019. Evaluation of citrus fiber as a natural replacer of sodium phosphate in alternatively-cured all-pork Bologna sausage. Meat Science. 157, 107883.

Rocchetti, G., Pellizzoni, M., Montesano, D. y Lucini, L. 2018. Italian Opuntia ficus-indica Cladodes as Rich Source of Bioactive Compounds with Health-Promoting Properties. Foods. 7(2), 24.

Saenz, C. 1997. Cladodes: a Source of Dietary Fiber. Journal of the Proffesional Association for Cactus Development. 2: 117-123.
Saenz, C., Berger, H., Corrales, J., Galletti, L., García, V., Higuera, I., Mondragón, C., Rodríguez, A., Sepúlveda, E. y Varnero, M. 2006. Utilización Agroindustrial del nopal. Boletín de Servicios Agrícolas de la FAO 162. FAO-AGST y FAOCACTUSNET, Roma.

Saenz, C., Yoong, M., Figuerola, F., Chiffelle, I. y María Estevez, A. 2012. Cactus pear cladodes powders as a source of dietary fibre: purification and properties. International Journal of Food Sciences and Nutrition. 63: 283-289.

Sánchez-Bell, G. D. 2006. Al nopal no sólo hay que verlo cuando tiene tunas. Cuadernos de nutrición. 29: 62-65.

Silva-Vazquez, R., Flores-Girón, E., Quintero-Ramos, A., Hume, M. E. y Mendez-Zamora, G. 2018. Effect of inulin and pectin on physicochemical characteristics and emulsion stability of meat batters. CyTA - Journal of Food. 16(1): 306-310.

Talukder, S. 2015 Effect of Dietary Fiber on Properties and Acceptance of Meat Products: A Review. Critical Reviews in Food Science and Nutrition. 55(7): 1005-1011.

Torres, J., Gonzalez-Morelo, K. y Acevedo-Correa, D. 2015. Análisis del Perfil de Textura en Frutas, Productos Cárnicos y Quesos. Revista ReCiTelA. 14: 63-75.

Van De Kamer, J. H. y Van Ginkel, L. 1952. Rapid determination of crude fiber in cereals. Cereal Chemistry. 29: 23-25.

Weemaes, C. A., Ooms, V., Van Loey, A. M. y Hendrickx, M. E. 1999. Kinetics of Chlorophyll Degradation and Color Loss in Heated Broccoli Juice. Journal of Agricultural and Food Chemistry. 47(6): 2404-2409.

Yadav, S., Pathera, A., Islam, R., Malik, A. y Sharma, D. 2018. Effect of wheat bran and dried carrot pomace addition on quality characteristics of chicken sausage. Asian-Australasian Journal of Animal Sciences. 31(5): 729-737.

Zhao, Y., Hou, Q., Zhuang, X., Wang, y Zhou, G. y Zhang, W. 2018. Effect of regenerated cellulose fiber on the physicochemical properties and sensory characteristics of fat-reduced emulsified sausage. LWT-Food Science and Technology. 97: 157-163. 